\title{
Gestión de estrategias para la prevención del estrés en el ámbito educativo: Un análisis desde la disciplina de Orientación
}

\author{
Strategies for stress management: an analysis from the discipline of Guidance
}

Recibido 15 enero 2014 • Aceptado 20 junio 2014 • Corregido 28 junio 2014

\author{
Wilbert Porras Quirós ${ }^{7}$ \\ Ministerio de Educación Pública \\ San José, Costa Rica \\ willrony@yahoo.es \\ Marianela Araya Marín ${ }^{2}$ \\ Ministerio de Educación Pública \\ Limón, Costa Rica \\ marianel09@gmail.com \\ Laura Fallas Fallas \\ Escuela Liceo María Auxiliadora \\ San José, Costa Rica \\ lauff 04@hotmail.com
}

\begin{abstract}
Resumen. La disciplina de Orientación en conjunto con la Dirección de los Centros Educativos, tiene como eje central de trabajo gestionar estrategias para disminuir el desarrollo de problemáticas personales y sociales en el ser humano, entre ellas el estrés, mediante la gestión de una educación integral, en la cual se fomente el uso de técnicas adecuadas para su manejo adecuado, con la finalidad de prevenir situaciones que pongan en riesgo la salud, generen bajo rendimiento y deserción estudiantil, entre otros. El presente estudio tiene como objetivo la exploración y abordaje de estrategias para el manejo del estrés utilizadas por un grupo de estudiantes de octavo año del Liceo Nocturno Alfredo González Flores, durante el 2012. El estudio se realiza mediante las premisas del paradigma naturalista y tiene un enfoque fenomenológico. Se concluye con la necesidad de profundizar la temática en la población, pues se evidencia en ella, múltiples agentes estresores por la particularidad de ser personas que son padres o madres de familia, quienes estudian y trabajan en su mayoría.
\end{abstract}

Bachiller y Licenciado en Orientación, Universidad Nacional. Labora actualmente como Orientador en el Colegio Técnico Profesional de La Gloria de Puriscal, el cual pertenece al Ministerio de Educación Pública de Costa Rica.

2 Bachiller y Licenciada en Orientación, Universidad Nacional. Se desempeña como Orientadora en la escuela de Excelencia San Bosco, La Rita, Pococí, en la provincia de Limón, perteneciente al Ministerio de Educación Pública de Costa Rica.

3 Bachiller en Orientación en la Universidad Nacional de Costa Rica. Se desempeña como Orientadora en una institución educativa privada, la Escuela Liceo María Auxiliadora, en San José, Costa Rica. \section{(C) $९ € \begin{aligned} & \text { Licencia Creative Commons } \\ & \text { Atribución-No-Comercial SinDerivadas } \\ & 3.0 \text { Costa Rica. }\end{aligned}$
EY NC ND}

Wilbert Porras Quirós, Marianela Araya Marín y Laura Fallas Fallas
Número publicado el 1 de julio del 2014 
Palabras clave. Estrés; técnicas para el manejo de estrés; orientación; gestión de la educación.

Abstract. The Guidance discipline and the schools' supervision, both of themfocused on prevention as the key to anticipate the development of personal and social problems. The main objective of this study is to explore strategies for stress management in a group of students from LiceoNocturno Alfredo González Flores. It is performed by the premises of the naturalistic paradigm and has a phenomenological approach. This work concludes with the need to examine the subject in this population, because there are multiple stress agents such as maternity, study and work among the students.

Keywords. Stress; techniques for stress management; guidance; education management

"Da tu primer paso ahora. No es necesario que veas el camino completo, pero da tu primer paso. El resto irá apareciendo a medida que camines"

Martin Luther, Jr.

\section{INTRODUCCIÓN}

Las instituciones educativas nocturnas enfrentan altos índices de deserción y repitencia, ocupando los primeros lugares en el Sistema Educativo en ese sentido, con un total de 35.414 casos hasta el 2013, según informes presentados por el Ministerio de Educación Pública de Costa Rica. Las razones por las cuales se da esta problemática, son multicausales; sin embargo, por las características de la población, el estrés es quizás una de las principales. Esto puede ser prevenido mediante intervenciones oportunas de profesionales en Orientación y con la colaboración oportuna del gestor o gestora educativa, pieza fundamental en el adecuado abordaje de este tipo de situaciones, ya que es un tema de relevancia académica, social y personal, pues atiende una temática que afecta el progreso del país -al ser la educación un eje fundamental de desarrollo-.

El presente artículo recopila los resultados obtenidos en intervenciones colectivas de Orientación que se han llevado a cabo con estudiantes de octavo año del Liceo Nocturno Alfredo González Flores, ubicado en la provincia de Heredia, durante el curso lectivo 2012, referentes a la temática de estrés y su contraste con la necesidad de realizar, evaluar y dar seguimiento a este tipo de actividades desde el planteamiento de la gestión educativa. El objetivo general fue explorar la concepción de estrés, así como los factores estresores y principales estrategias utilizadas por estas personas para su adecuado abordaje, a partir de la presentación de resultados obtenidos de las intervenciones grupales - partiendo de los resultados del diagnóstico- en las cuales se propuso como objetivo dar a conocer técnicas y estrategias para el manejo adecuado del estrés, con el fin promover la calidad de vida en las personas participantes. 
Rev. Gestión de la Educación, Vol. 4, N² 2, [131-149], ISSN: 2215-2288, julio-diciembre, 2014

DOI: http://dx.doi.org/10.15517/rge.v4i2.15148

URL: http://revistas.ucr.ac.cr/index.php/gestedu

Es importante resaltar que, desde la gestión educativa, es fundamental la utilización de todos los recursos con los cuales se cuenta en las instituciones educativas -entre ellos el recurso humano- para fortalecer planes de intervención donde se busque mejorar la calidad de vida del estudiantado en aspectos tan complejos como lo es, actualmente, el estrés en la sociedad. Desde la administración de un centro educativo, al visualizar la necesidad de fomentar el desarrollo integral de los seres humanos, se hace trascendental promover espacios de reflexión, en conjunto con profesionales de ayuda como lo son los orientadores y orientadoras, donde se aborden temáticas del acontecer diario y se propongan soluciones viables para afrontarlas de una manera adecuada.

Actualmente, una gestión educativa visionaria conlleva el uso de los preceptos de la educación permanente, en la cual se hace necesario "una gestión que articule la visión de lo educativo-cultural y que lidere procesos de comunicación con una visión holística e integral de las áreas de gestión directiva, académica-pedagógica, administrativa-financiera y de convivencia y comunidad" (Correa de Urrea, Álvarez y Correa, s.f., p. 4). Aunado a una visión humanista de la población a la cual se atiende, percibiéndoles como personas con necesidades y fortalezas que merecen una atención especializada de calidad, mediante el acompañamiento profesional oportuno.

La gestión educativa demanda la articulación de esfuerzos desde todo lo amplio de su estructura, procurando que el estudiantado logre una educación donde se aborden los contenidos propios del modelo académico, enriquecido con aprendizajes prácticos para la vida, en los cuales, más allá de la alfabetización y la educación aritmética básica, se fomente la capacidad para desenvolverse en este mundo, pues en la actualidad, se requiere una amplia variedad de competencias cognitivas, sociales y prácticas (Fondo de las Naciones Unidas para la Infancia [UNICEF], s.f.), producto de las múltiples problemáticas sociales tales como la presión grupal, la escases de recursos económicos, fuentes de empleo inestables, entre otros, con el fin de alcanzar la autorrealización, donde el estrés generado por estas eventualidades, más que un agente negativo se convierte en un propulsor de energía básica para la consecución de las metas propuestas en el proyecto de vida de cada persona, como se abordara posteriormente.

De esta forma, la gestión educativa debe colaborar con el adecuado manejo del estrés en la población estudiantil, mediante la creación de espacios para tal fin, acompañado de la intervención de profesionales preparados para ello, en el ámbito de la orientación; es especialmente importante como estrategia para la prevención de problemáticas tan fuertes como la deserción y la repitencia escolar, que suelen ser la respuesta a no contar con las técnicas necesarias para manejar el estrés de manera adecuada.

El objetivo de realizar procesos de intervención en la población estudiantil, propiamente en temáticas como el manejo de estrés, es lograr que ellos y ellas alcancen las herramientas disponibles para que puedan enfrentar los factores estresantes de una manera positiva para

Licencia Creative Commons 
su vida, no esquivando o reprimiendo su sentir ante diversas situaciones, sino más bien siendo conscientes de que son parte de la vida y por ello, se deben enfrentar positivamente.

Cabe resaltar la necesidad de fundamentar el accionar de la gestión educativa en un modelo que retome y resignifique el papel de las personas en las organizaciones, desde una perspectiva humanista, social y cultural de la administración, mediante el establecimiento del compromiso de la participación del colectivo y de la construcción de metas comunes, donde se asuma con responsabilidad y liderazgo el desarrollo de acciones pertinentes para lograr alcanzar la autorrealización de todos aquellos seres humanos vinculados con el proceso de enseñanza aprendizaje, logrando sobrellevar situaciones como el estrés, producido por las actividades que se llevan a cabo en este contexto (Correa de Urrea et al., s.f.).

\section{REFERENTES TEÓRICO-CONCEPTUALES}

\section{Estrés}

En la actualidad, el término estrés es comúnmente usado por casi todas las poblaciones, sin distinguir etapa del desarrollo, condición económica o estatus social. Dentro del sistema educativo esta realidad se mantiene, vinculándose al fracaso y bajo rendimiento escolar (Zeidner, 1998; McDonald, 2001).

Cabe resaltar, según Naranjo (2009) que:

Todas las personas necesitan aprender a prevenir y controlar el estrés. Quien no lo hace, puede poner en peligro su salud y su tranquilidad, mientras que quien conoce y pone en práctica acciones adecuadas para prevenirlo y afrontarlo puede disfrutar de un estilo de vida más sano y más satisfactorio. (p. 171)

En ello radica la importancia de sensibilizar a la población estudiantilal respecto, buscando crear conciencia de los beneficios en la salud integral al conocer técnicas y estrategias para su prevención, esto como una herramienta fundamental para fortalecer procesos de autoconocimiento en donde impere la constancia -y quizás- una estrategia más para afrontar problemáticas tan severas en las instituciones educativas nocturnas, como lo son la repitencia, la deserción, el ausentismo y la desmotivación, aspectos claves para ser incluidos en la gestión educativa.

Un referente para abarcar la temática de estrés es Selye y los estudios realizados con sus pacientes (Sandín, 2002; Barraza, 2007; Berrío y Mazo, 2011); de ellos, se marca la necesidad de abordar en el ser humano, aspectos que trascienden meramente el plano fisiológico. Para 1930, Berrío y Mazo (2011) destacan que "los enfermos a quienes observaba, indiferentemente de la enfermedad que sufrían, tenían síntomas comunes y generales como agotamiento, pérdida del apetito, baja de peso, astenia, entre otros síntomas inespecíficos" (p. 67), dando el inicio del

Licencia Creative Commons

Atribución-No-Comercial SinDerivadas (cc) (†) $\$$ 3.0 Costa Rica. 
Rev. Gestión de la Educación, Vol. 4, N² 2, [131-149], ISSN: 2215-2288, julio-diciembre, 2014

DOI: http://dx.doi.org/10.15517/rge.v4i2.15148

URL: http://revistas.ucr.ac.cr/index.php/gestedu

estudio de un tema, el cual ha sido visto como un problema de salud pública, pues tiene un gran impacto en la vida cotidiana de todos los seres humanos en los diferentes contextos en donde se desarrolle, incluyendo el educativo.

Al buscar una definición de estrés, se llega a una de las situaciones muy comunes de las ciencias sociales al querer definir un concepto, su basta complejidad; es por ello, que "(...) el estrés no puede definirse desde una concepción unívoca, sino que debe comprenderse como un conjunto de variables que funcionan de forma sincrónica y diacrónica, dinamizadas a lo largo del ciclo vital (...)" (Berrío y Mazo, 2011, p. 68).

El estrés se puede ver como la respuesta de un organismo ante múltiples situaciones que le afectan positiva o negativamente, produciendo su actuar; en el sistema educativo se ve fácilmente vivenciado por la población estudiantil durante la elaboración de pruebas escritas y orales, donde por su inadecuado manejo suele repercutir en resultados incongruentes con el tiempo y los esfuerzos realizados para la comprensión de los temas abordados en clases. El estrés es una fuerza, cuyo fin, es permitir la movilidad mediante una energía intrínseca e extrínseca con repercusiones visibles para la persona.

Según Bautista y Ré (2007), el estrés"... es la reacción / respuesta tensional de la persona a través de todas y cada una de sus dimensiones" (p. 47); está implícito en todos sus quehaceres, afectándole constantemente el área física, social, emocional, cognitiva, espiritual, vocacionalacadémica. Además, puede ser incrementado por aspectos como la carga académica y su calidad de las relaciones humanas, especialmente en ambientes frecuentados en forma regular como lo son centros educativos.

Así pues, el estrés si no es manejado adecuadamente por las personas se puede convertir en una fuente de vulnerabilidad, especialmente en el estudiantado. Por lo tanto, desde una gestión de la educación visionaria y preocupada por el bienestar de la población estudiantil, se deben contemplar las estrategias pertinentes para evidenciar los principales factores estresores vivenciados por ellos y desarrollar, de esta manera, esfuerzos para fortalecerles técnicas y estrategias para su adecuado manejo.

\section{Tipos de estrés}

Existen diferentes clasificaciones para el estrés; algunas van a depender del grado de intensidad experimentado, otras de las repercusiones ocasionadas en la persona. De acuerdo con Selye (1956), mencionado por Landy y Conte (2005), se clasifican básicamente de dos maneras: la primera, es el estrés positivo -conocido en inglés como eustress- cuyo fin es producir retos en la persona de donde se obtiene la energía para proponerse y alcanzar metas; la segunda, corresponde al llamado estrés negativo -en inglés se conoce como distress- caracterizado por ser una situación estresante, la cual perdura en el tiempo y tiene consecuencias nocivas para la salud.

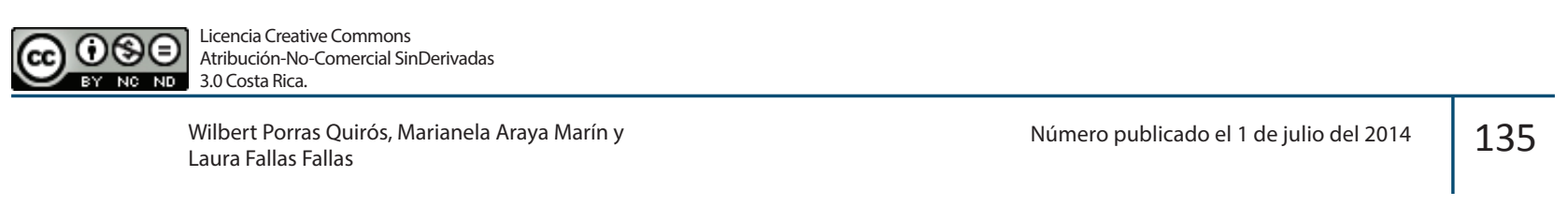


Otra de las formas más reconocidas del estrés, mencionada por Sandín (2002), es el estrés crónico, el cual:

... consiste en problemas, amenazas y conflictos relativamente duraderos que la mayor parte de la gente se encuentra en sus vidas diarias. Muchos de estos estresores crónicos se relacionan con los principales roles sociales, y consisten, por ejemplo, en dificultades en el trabajo, problemas de pareja o complicaciones en las relaciones entre padres e hijo. (p. 147)

Asimismo, se destaca el estrés por sucesos menores que hace referencias a situaciones de la cotidianidad, en donde se resalta:

... la acumulación de experiencias negativas aparentemente de poca importancia: en el curso de un día una persona puede, por ejemplo, discutir con su esposo/a, llegar tarde a una cita importante, ser mal atendida en el supermercado, tener problemas para aparcar el coche, no encontrar un documento importante y quedarse temporalmente sin dinero ... (Sandín, 2002, pp. 149-150)

Se reconocen más clasificaciones de estrés que las mencionadas anteriormente; sin embargo, por los propósitos del presente escrito, se deja a conciencia de la persona lectora la necesidad de profundizar -si es de su interés- en la temática. Lo fundamental es conocer su existencia para desarrollar estrategias de prevención que beneficien al estudiantado.

Ahora bien, dentro de las instituciones educativas, desde su gestión, se hace importante reconocer la existencia del llamado estrés académico, el cual se origina dentro del contexto educativo por la combinación de la carga académica con aspectos como la familia, el trabajo, el tráfico, entre otros, lo cual agota las reservas del estudiante, repercutiendo en una serie de consecuencias como diferentes enfermedades de toda índole, repitencia y desmotivación escolar (Pulido, Serrano, Valdés, Chávez, Hidalgo y Vera, 2011).

Se resalta que este tipo de estrés aumenta conforme el estudiante vaya subiendo de nivel académico, por las cargas de trabajo que se intensifican y dificultan conforme se pasa de grado, pero también porque coinciden con etapas de la vida en la que el ser humano debe enfrentarse a muchos cambios, tales como separación o mayor autonomía de la familia, la incorporación al mercado laboral, la conformación de una pareja, la adaptación a un medio poco habitual como suele ser la secundaria, en donde existe una mayor población estudiantil, así como del profesorado (Pulido et al., 2011). Estos hechos producen crisis circunstanciales y del desarrollo (Fernández, 2010), las cuales es necesario reconocer para su abordaje en las gestiones propias del actuar institucional de calidad.

\section{Factores estresores}

El estudiante, especialmente aquel que es partícipe de la modalidad académica nocturna, se ve expuesto a múltiples factores estresantes -posiblemente más que cualquier otro estudiante

Licencia Creative Commons

Atribución-No-Comercial SinDerivadas (cc) (†) 3.0 Costa Rica. 
Rev. Gestión de la Educación, Vol. 4, N² 2, [131-149], ISSN: 2215-2288, julio-diciembre, 2014

DOI: http://dx.doi.org/10.15517/rge.v4i2.15148

URL: http://revistas.ucr.ac.cr/index.php/gestedu

de educación diurna-, los cuales afectan su vida y repercuten en su formación académica; ante ello, la necesidad de gestionar esfuerzos desde la institución para contrarrestarlos mediante una educación integral que potencie el autoconomiento y la conformación de herramientas preventivas del estrés negativo.

Ahora bien, la forma en cómo cada persona reacciona ante el estrés va a depender de las herramientas adquiridas por ella durante todo el ciclo vital y la intensidad de los estresores (o sea, lo que genera el estrés). De acuerdo con Selye (1956), citado por De Mézerville (2004, p. 217), estos son clasificados como: "Estresores normales: Necesidades y demandas de la vida diaria; Estresores especiales: Cambios evolutivos y periodos transicionales; Estresores extraordinarios: Situaciones de pérdida y experiencias traumáticas".

Los factores estresantes, así como la forma de sentir el estrés, varían de una persona a otra, resaltando que:

... las condiciones sociales (organización social, apoyo social, aspectos socioeconómicos, estatus marital, rol laboral, género, etc.) pueden estar implicadas tanto en el origen como en las consecuencias de las experiencias estresantes. Las situaciones sociales pueden provocar altos niveles de estrés que, a su vez, afectan a la salud, a la calidad de vida y la longevidad. (Cockerham, 2001, citado por Sandín, 2002, p. 143)

En lo anterior, radica la importancia de gestionar, desde los modelos de educación, consciencia en las personas acerca de sus estilos de vida y en cómo estos -muchas veces- son afectados por las actividades realizadas, especialmente en poblaciones como la que asiste a la modalidad académica nocturna, las cuales combinan trabajo, familia y la asistencia al centro educativo, enfrentando así las dificultades propias de la carga: académica (horarios, roces con profesores, grupo de estudio...); familiar (cuido de la progenie, labores domésticas, pareja...); laboral (jefatura, normativas, horarios..) y personal (crisis del desarrollo, circunstanciales, vocacionales), aunado a la carencia de estrategias adecuadas para el manejo de estrés producido, eventualmente, en cada una de estas dimensiones.

Cabe resaltar que el entorno suele constituir un estresor más para el individuo, según los diferentes elementos poseídos en él, así como la interacción dada entre ellos y las características básicas de ambos. Se hace evidente, dentro de la cotidianidad, la existencia de algunos contextos potencialmente generadores de estrés, los cuales promueven en la persona -desde sus valoraciones cognitivas- el rechazo o la resistencia a enfrentarlos, aún si por condiciones como estudio o trabajo lo deben hacer, convirtiéndose en un estresor perjudicial para su salud (Barraza, 2010). Asimismo, el autor menciona que "un entorno potencialmente generador de estrés es un entorno novedoso en sus demandas, exageradamente demandante, altamente impredecible, escasamente controlable, con demandas de alta duración y elevada intensidad..." (p. 1). 
Para algunas personas que asisten a centros educativos nocturnos, suele existir resistencia para su asistencia regular, esto por la necesidad de una llegada pronta a su hogar para estar en compañía de su familia, máxime después de un día laboral; sin embargo, desde intervenciones de profesionales en orientación, en conjunto con la dirección del centro educativo, se pueden desarrollar estrategias que posibiliten ver en la institución, un lugar donde se logre un crecimiento integral, el cual incentive un aumento en la calidad de vida de aspectos vitalicios como lo es el manejo adecuado del estrés.

Si bien es cierto, cada institución educativa tiene sus particularidades, la modalidad académica nocturna, por las características propias de la población atendida (padres y madres de familia, gente trabajadora en su mayoría), demanda de una gestión enfocada en apoyo y contención de sus necesidades, debido a su gran diversidad. Además, esta posee como finalidad disminuir el fracaso escolar y posibilitar el acceso con equidad al Sistema Educativo Costarricense (Ministerio de Educación Pública, 2008). Ante ello, como parte de la gestión administrativa de estos centros, se deben estar realizando constantemente diagnósticos de los emergentes vivenciados por esta población, permitiendo así, trabajar temáticas como el estrés para generar planes preventivos que posibiliten la atención pronta y oportuna de las situaciones que les genere esta problemática, con el fin de ofrecerles una educación de calidad, sin dejar de lado las necesidades específicas, centrada en el bienestar integral de cada uno de sus estudiantes.

\section{Técnicas para el manejo del estrés desde la gestión educativa para el mejoramiento de la calidad de vida}

El manejo del estrés posibilita, en los seres humanos, la adquisición de herramientas capaces de impulsar estilos de vida saludables, los cuales generan en las persona la integración de las áreas emocional, física, intelectual, espiritual, vocacional-académica, entre otras, en pro de implementar la cosmovisión del ser humano y su autorrealización. Dentro de la gestión educativa, como parte de los procesos de enseñanza académica y para la vida, se debe contemplar el desarrollo integral de la persona como uno de los objetivos prioritarios; por ende, la necesidad de promover el conocimiento y vivencia de técnicas, las cuales nutran el "eustress" o estrés positivo, logrando procesos educativos más conscientes y sensibles de las necesidades del estudiantado.

Algunas de las técnicas para el manejo del estrés, implementado dentro de las intervenciones colectivas realizadas, se mencionan seguidamente:

La abrazoterapia: en la vida cotidiana se es partícipe de lo positivo que resulta un abrazo en casi cualquier situación; estos generan seguridad, brindan apoyo, dan contención y favorecen al fortalecimiento de las relaciones interpersonales y a la generación de factores protectores para superar cualquier adversidad. Se pueden recibir de otra persona o incluso, se puede dar un autoabrazo, en donde se generan energías y contención consigo mismo.

Licencia Creative Commons 
Esta es una técnica que, a partir de acciones tan sencillas pero tan eficaces como son los abrazos, nutre al individuo de elementos que le permiten disfrutar plenamente del momento, en un acto más allá de lo físico. Según De Mézerville (2012):

Los abrazos, además de hacernos sentir bien, se emplean para aliviar el dolor, la depresión y la ansiedad. Provocan alteraciones fisiológicas positivas en quien toca y en quien es tocado. Acrecienta la voluntad de vivir a los enfermos. Es de todos bien sabido que cuatro abrazos al día son necesarios para sobrevivir, ocho para mantenerse y doce para crecer como persona. (párr. 4, Abrazoterapia)

El humor: ya sea dentro de un grupo de amigos o al conocer una persona, el humor es una herramienta que posibilita crear espacios de vinculación y generar un clima de confianza entre el grupo. Al respecto, Rose (2002) menciona que:

Además de ser divertido, reír es un buen ejercicio para el corazón y los pulmones. Asimismo, según ciertas investigaciones, reír también contribuye a reducir la presión arterial, relaja los músculos, alivia el dolor, reduce las hormonas del estrés y dispara el sistema inmunológico, aumentando la producción de células destructoras de enfermedades. La risa es un detonante para la liberación de endorfinas, los analgésicos naturales del cuerpo, y producen una sensación general de bienestar. (p. 33)

El humor permite sobrellevar situaciones agobiantes, aumenta el optimismo y la visión de una vida con más goce y disfrute pleno, enriquece la promoción de relaciones interpersonales sanas y productivas; además, le inyecta una chispa de positivismo a cualquier tarea a realizar. Las personas pueden ser más felices si sonríen con frecuencia, posibilitando vivir más años, así como el desarrollo de una autoestima más adecuada.

Los masajes: esta técnica favorece a la liberación de endorfinas, sustancia que posibilita sentir bienestar, así como prevenir y combatir los dolores fisiológicos (De Mézerville, 2012). Es algo a lo cual se debe recurrir no solo por enfermedad, sino que debe ser parte de un plan preventivo de enfermedades, el cual trasciende lo físico y conlleva a entrar en un plano psicoespiritual. Rose (2002) menciona que:

Utilizar el tacto es una manera muy efectiva de relajarse, y el masaje, en particular, es una de las formas más fiables de aliviar el estrés y relajar los músculos doloridos. El masaje es una terapia sedante que libera tensiones y reduce la ansiedad... (p. 16)

Este puede ser acompañado de musicoterapia y aromaterapia, con la finalidad de lograr mayor contacto interno de la persona a quien se le realiza o que por su propia cuenta lo hace.

La musicoterapia: incrementa o disminuye la energía muscular, permite acelerar la respiración o alterar su regularidad favoreciendo cambios en el pulso, la presión sanguínea y la función endocrina (Benenzon, 1985, citado por Peraza y Zaldívar, 2003). Además, disminuye 
el impacto de los estímulos sensoriales de diversas formas, se tiende a reducir o demorar la fatiga e incrementa el endurecimiento muscular, aumenta la actividad, incrementa la extensión de los reflejos musculares empleados en escribir, dibujar, entre otras. Finalmente, es capaz de provocar cambios en el metabolismo y en la síntesis de variados procesos enzimáticos (Peraza y Zaldívar, 2003).

Entre los efectos psicológicos generados por la musicoterapia, se señala como el más importante el comunicativo, pues se posibilita convertir a las defensas que surgen en el lenguaje verbal en medios de expresión emocional, para lo cual es necesario tener en cuenta los significados que poseen los diferentes elementos que componen la música, entre ellos la armonía, el ritmo, la melodía, el timbre, la frecuencia, la intensidad y el volumen, de tal forma que se combinan, para de esta manera lograr cambios en los estados de ánimo (Roca, s.f.).

La cromoterapia: se apoya en los preceptos de la teoría de colores. Para Rose (2002):

... los colores pueden tener un profundo efecto en su estado de ánimo, vitalidad y bienestar. El color amarillo y el rojo son estimulantes; el azul y el verde, tranquilizantes. Los terapeutas del color utilizan colores diferentes para mejorar la salud física, emocional y espiritual de sus pacientes, generalmente aplicando luces de distinta tonalidad sobre sus cuerpos. (p. 27)

La elección de los colores no es algo al azar, sino que depende del momento y el estado anímico de las personas; según la teoría, responde al aquí y ahora, pues son una de las manifestaciones de las emociones, en donde se representa sensaciones y situaciones, basadas en la observación y la experiencia.

La cromoterapia es un método de armonización y de ayuda a la curación natural de ciertas enfermedades mediante el uso de los colores, los cuales corresponden a vibraciones que tienen velocidades, longitudes y ritmos de ondas diferentes. Estos ejercen una influencia física, psíquica y emocional imperceptible de una manera consciente al ser humano y permite a la energía vital tener un estado que facilita la auto sanación (Carrillo, 2010).

La cromoterapia se aplica principalmente mediante el uso de mandalas, arte milenario que permite por medio de un soporte gráfico llegar a la relajación, la concentración y a la meditación; además, posibilita expresar el pensamiento propio con naturaleza y creatividad; estas son representaciones geométricas y simbólicas del universo y de sí mismo que significan rueda, corona, círculo, rotación. Estas son utilizadas, desde tiempos remotos, en todos los países del mundo: India, China, Tibet, pueblos originarios de América, Australia, África, entre otros (Pedagogía 300, s.f.).

La aromaterapia: es una de las técnicas más reconocidas a nivel mundial para el control del estrés. Según De Mézerville (2004) tiene su origen en la cultura oriental muy antigua, cuyo

Licencia Creative Commons

Atribución-No-Comercial SinDerivadas (cc) (†) 3.0 Costa Rica. 
Rev. Gestión de la Educación, Vol. 4, N² 2, [131-149], ISSN: 2215-2288, julio-diciembre, 2014

DOI: http://dx.doi.org/10.15517/rge.v4i2.15148

URL: http://revistas.ucr.ac.cr/index.php/gestedu

efecto es relajar. Consiste en utilizar aceites de hierbas y otras plantas aromáticas para que, aplicadas en el cuerpo, logren la relajación o el alivio de un dolor o trastorno.

La hipnoterapia: “... es un proceso por el cual se ayuda a la gente a utilizar sus propias asociaciones mentales, recuerdos, y potenciales de vida, para lograr sus propias metas terapéuticas" (De Mézerville, 2004, p. 3). Se puede completar con la autohipnosis, en la cual la persona va logrando, por sí misma, entrar a un estado de relajación mediante la evocación de imágenes, cuentas regresivas o historias progresivas para inducir la tranquilidad.

Ejercicios de respiración: generalmente se ve como algo inherente al ser humano que se hace involuntariamente, pero cuando es controlado y se concientiza, se cambia totalmente la percepción de relajación obtenida. Es recomendable hacer ejercicios de respiración diariamente, durante las primeras horas de las mañanas y practicarla constantemente durante el resto del día.

Las técnicas propuestas anteriormente, mencionadas para el manejo y prevención del estrés, son de sencilla implementación para las personas; responden a hacer conscientes muchos de los actos realizados a diario; sin embargo, pueden ayudar a que las personas adquieran valiosas herramientas, las cuales pueden mitigar el efecto de situaciones adversas para su vida.

Desde una gestión administrativa institucional eficaz, se pueden promover planes de prevención en donde, en conjunto con el personal docente y administrativo, se permita la vivencia de espacios de relajación en los que se implementen estas técnicas, con la finalidad de promover la calidad de vida de los individuos que integran el sistema educativo.

\section{METODOLOGÍA}

El presente estudio se realizó mediante las premisas del paradigma naturalista, el cual se caracteriza por posibilitar la comprensión y significado de la realidad social de los seres humanos, dando la posibilidad de estudiar el fenómeno en su contexto natural, teniendo presente diversos aspectos capaces de afectar a la persona participante (Barrantes, 2006). También, se implementan aspectos del enfoque fenomenológico, con la finalidad de presentar la realidad tal y cual las personas implicadas la vivencian.

La población del estudio, se caracteriza por su diversidad, entre ellas sobresale: diferencias entre la edad de sus integrantes, ya que oscilan entre los 18 a 35 años aproximadamente; existen amas de casa, madres y padres de familia; la gran mayoría tienen un trabajo fijo y asisten por las noches, de lunes a viernes, de 6: 00 pm. a 9: 45 pm. a estudiar, posterior a un día normal de trabajo.

Los instrumentos de investigación aplicados fueron la observación, el grupo focal y la elaboración de la técnica collage, en donde las personas escribieron los factores estresores que les afectan y los principales métodos utilizadas por ellas para darle un adecuado manejo al 
estrés. Por ser una investigación de tipo naturalista, los resultados obtenidos se presentan de una manera narrada y detallada, de acuerdo con lo expresado por las personas participantes del estudio.

\section{RESULTADOS DEL DIAGÓSTICO}

Los resultados se describen seguidamente, partiendo de la información obtenida por parte de las personas participantes y es ordenada de acuerdo con los intereses propios de la investigación realizada.

\section{Concepción de estrés de las personas integrantes de la sección 8-4 del Liceo Nocturno Alfredo González Flores}

Las personas participantes tienen una concepción negativa del estrés. Es una fuerza -según ellas- que surge a partir de las presiones generadas en el contexto del cual son partícipes y es producido por diferentes situaciones, entre ellas: la familia, el trabajo y el centro de estudio. Se rescata que, la mayoría de las personas, tienen una posición construida con respecto a la temática, es algo conocido para ellos; sin embargo, coinciden en darle un carácter meramente negativo.

Algunas de las expresiones empleadas por las personas participantes para definir estrés son:

- "Es lo que siento cuando algo me está causando preocupación y lo siento en el cuerpo, especialmente en los hombros";

- "Es lo que me produce el jefe, por estarme mandando a toda hora";

- "Es el resultado de pensar en los exámenes y los trabajos extraclase";

- "Es lo que me produce mi pareja cuando llega con sus amistades, además al cuidado de los hijos y a tener que ir a trabajar y luego al colegio";

- "Es lo que me genera estar haciendo las tareas de la casa, del colegio, tener que pensar en cuidar a los hijos y pensar en ayudarles a hacer sus tareas de la escuela, es la razón por la que no me gusta estudiar, es lo que hace que en los exámenes no me pueda concentrar y obtenga un mal resultado, es lo que siento al matricularme a principio de año, sabiendo que cada día tengo que estudiar más y tengo menos tiempo para ello, es lo que me genera estudiar antes de los exámenes". (Comunicaciones personales, 2012)

Como se puede observar, el estrés es visto como una energía meramente negativa para el estudiantado; sin embargo, se coincide en que es la fuerza que les permite actuar. Por lo tanto, como lo menciona Naranjo (2009), lo importante es empezar a prevenir sus repercusiones negativas, fortaleciendo estilos de vida sanos y satisfactorios en la población, pues si no podría influir negativamente en la motivación para asistir a la institución educativa.

Es importante destacar que, desde la gestión educativa, es una necesidad imperante el

Licencia Creative Commons 
Rev. Gestión de la Educación, Vol. 4, N² 2, [131-149], ISSN: 2215-2288, julio-diciembre, 2014

DOI: http://dx.doi.org/10.15517/rge.v4i2.15148

URL: http://revistas.ucr.ac.cr/index.php/gestedu

manejar conceptos relacionados con estrés y las formas de abordarlo, ya que, basados en el adecuado conocimiento y la disposición positiva de crear espacios y brindar atención oportuna, es posible conseguir resultados beneficiosos en la población educativa.

\section{Principales factores estresores de las personas integrantes de la sección 8-4 del Liceo Nocturno Alfredo González Flores}

Los principales factores que afectan a la población participante, se subdividen por áreas para facilitar su entendimiento. A saber:

Estresores familiares: sobresalen como estresores los conflictos maritales o de pareja, con los hijos y la realización de las labores de la casa, las cuales son vinculadas a creencias machistas en donde la mujer debe realizar la mayoría de las tareas, sin contar con el apoyo de su pareja, no se cuenta con tiempo suficiente para compartir, distanciamiento en las relaciones de pareja, la indisciplina de los hijos e hijas, las tareas de los hijos e hijas, roles demarcados para hombres y mujeres en relación con el cuido de la progenie y la atención de sus quehaceres escolares, distanciamiento del hombre de las tareas domésticas, problemas con las amistades de la pareja.

Como lo menciona Cockerham (2001), citado por Sandín (2002), el estrés difiere de situaciones particulares de cada ser humano. Se pueden observar diferencias acarreadas por el género y el estado civil de las personas. En su mayoría, las mujeres vivencian mayor estrés debido a las tareas domésticas, así como el machismo y los hombres, relacionado con la relación de pareja.

Estresores laborales: el trabajo es considerado el principal estresor, debido, en su mayoría, a diferencias con la jefatura -en específico, los problemas que se tienen con ellos- pues dicen ser muy abusivos en la utilización del poder sobre las personas subalternas, convirtiéndose en un detonante para llegar con estrés a la casa y principalmente, al centro educativo (lugar en donde se suele hacer catarsis de los problemas tenidos con el grupo de compañeros y compañeras).

Con mayores responsabilidades, más cantidad de estrés, en ello coincide la población atendida. En este aspecto, se marca una diferencia entre quienes laboran y aquellos que no lo hacen. El primer grupo ve en no trabajar, una posibilidad para tener más tiempo para sus estudios, prescindiendo del estrés que produce tener una jefatura y las responsabilidades que ello implica. El segundo grupo comenta que no contar con una fuente laboral les genera mucho estrés por carecer de fuentes de ingreso, teniendo que depender de la familia u otras personas.

En el ámbito laboral, se evidencian diferencias asociadas al género, en el sentido de reconocer las tareas domésticas como uno de los oficios más difíciles, el cual no tiene remuneración y no se le da la importancia que requiere, siendo meramente asociado con la mujer. El estrés producido por el ámbito laboral es el que más repercusiones tiene en el ámbito 
educativo, pues influye directamente en estudiar o dejar de hacerlo, ya que produce mucho desgaste físico y emocional, agravado por el hecho de no contar con espacios donde hacer procesos catárticos, así como desconocer técnicas para manejar el estrés producido en estos espacios.

Estresores académicos: en este punto sobresalen las pruebas académicas (estudiar y no lograr tener una buena calificación), el ruido constante en la institución de estudio producto de la ubicación geográfica, diferencias con los profesores (algunos no muestran motivación por enseñar o carecen de empatía), los promedios bajos, no llegar a tiempo o incluso, ausentarse por eventualidades laborales o familiares, la carga académica, los trabajos extraclase, el temor a reprobar por discontinuidad en los estudios o dificultades de aprendizaje presentadas en instituciones en las cuales se ha estado anteriormente, diferencias con los compañeros y compañeras, el horario, no tener dinero para cubrir el costo de los materiales solicitados y finalmente, el constante aumento de la carga académica.

Para la población participante, por sus características particulares, es en la parte académica donde tiene mayores repercusiones los estresores producidos en la familia y el ámbito laboral, ya que llegan desconcentrados al centro educativo y por horario, muchas veces, es donde se concentra todo el estrés del día, generando el deseo de desertar por cansancio; sin embargo, esa misma presión que les generan estos espacios, es lo que les impulsa a luchar para hacer un cambio en sus vidas, garantizándose un posible cambio, en el futuro, de las condiciones laborales actuales. Además, la familia, aunque coinciden que genera mucho estrés, también es la inspiración que permite superar muchos de los obstáculos tenidos, para garantizarles una mayor calidad de vida.

Otros estresores: ir al hospital acompañando a un miembro de su familia o en caso de quebrantos de su salud, las filas generadas en el cajero, situaciones sentimentales, tener dificultades para dormir, madrugar, exceso de trabajo, las deudas, no tener dinero, situaciones incomodas y emergentes, transportarse en bus, las presas, la impaciencia, los chismes, las "peleas".

Algunas de las expresiones externadas por las personas participantes del estudio son:

- "Me siento muy estresado cuando tengo que andar todo el tiempo escuchando al jefe repetirme lo mismo";

- "Mi esposo me estresa demasiado, máximo cuando llega con sus amigos a la casa y no me ayuda ni a recoger un plato";

- "Hay profesores a los que no les entiendo nada y eso me hace sentir incomoda en clases, hasta tal punto de no querer venir";

- "Me siento muy preocupada tener que pensar en todo lo que debo pagar y no tener plata para hacerlo".

Licencia Creative Commons 
Si bien es cierto, la población del estudio cuenta con múltiples estresores, muchos de ellos ven en estos la fuerza que les motiva a seguir adelante; sin embargo, reconocen la carencia de técnicas y estrategias para mitigar los efectos del estrés producidos por las actividades que realizan, sintiendo la necesidad de tener un mayor acompañamiento en espacios como la institución educativa, con el fin de dar un manejo adecuado a la problemática.

Es por esto, que se resalta la importancia de la gestión educativa en este tipo de circunstancias, ya que sin la ayuda oportuna del gestor o gestora del centro educativo, se imposibilita la adecuada intervención y por tanto, la permanencia de los estudiantes en la institución.

\section{Técnicas utilizadas para el manejo del estrés por los y las integrantes de la sección 8-4 del Liceo Nocturno Alfredo González Flores}

Las principales técnicas utilizadas por las personas involucradas en la investigación son: asistir a servicios religiosos, compartir con la familia, salir a pasear o comer con el grupo de amigos, jugar PlayStation, respirar profundo, pegarle a la pared, hablar con otras personas, contar hasta diez, hacer deporte, omitir escuchar "chismes", escuchar música, llorar, reírse, pensar en algo bonito, maldecir, bailar, hacer ejercicio, hacerse masajes, tomar café, llorar, acercarse a la naturaleza, fumar, ver películas con la familia, asistir al colegio, nadar, compartir con los compañeros y compañeras del colegio.

Se rescata la coincidencia de la mayoría de los participantes, en la necesidad de reforzar las técnicas para el manejo de estrés, pues mencionan tener dificultades para hacerlo, ya que reciben muchas presiones propias del contexto en donde se desenvuelven y la falta de información de ellas, debido a que en realidad no las conocen (solo las han escuchado mencionar); esto les ha llevado, en muchos momentos, a querer desertar de estudiary tener problemas en el trabajo, así como en la familia.

\section{Resultados de las intervenciones colectivas de técnicas para el manejo y prevención del estrés}

El grupo se caracteriza por ser muy diverso, por lo que implicó desarrollar estrategias que posibilitaran mayor integración; eso se convirtió en todo un reto para realizar la intervención, así como en una herramienta para integrar diferentes biografías, las cuales posibilitaran la apertura al diálogo y el compartir historias de vida, en función de las estrategias utilizadas por ellos y ellas para prevenir, así como dar un manejo más adecuado del estrés.

Se evidenció mucha motivación, por parte de la población, en participar de las actividades realizadas y la necesidad de continuar con este tipo de intervenciones, de tal manera que se les posibilite la construcción de herramientas para dar un adecuado manejo del estrés y de esta manera, mejorar su calidad de vida.

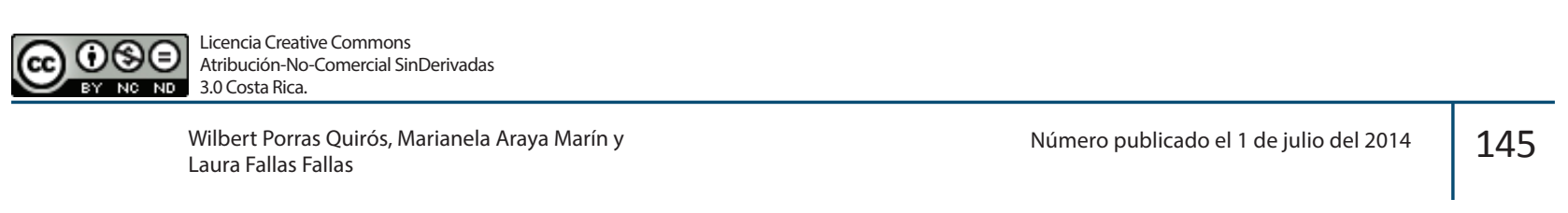


Muchas personas comentaron que, desde hace ya muchos años, no dedican tiempo para sí mismas por las carreras propias de la vida cotidiana, viendo la repercusión de tal acción en la familia, el trabajo y el ámbito educativo. Reconocen que muchos de los problemas interpersonales dados en el salón de clases son producidos por el estrés de sus integrantes. Además, si contaran con más apoyo para relajarse, incluso antes de un examen, los resultados variarían positivamente y eso se reflejaría en la estancia en el centro educativo.

Es por esto, que la gestión educativa tiene un papel importante, ya que es desde ahí que se propician actividades y proyectos en miras a mejorar la calidad educativa de la población estudiantil que presenta estas características.

\section{CONCLUSIONES}

Desde una visión integral del ser humano, es necesario gestionar esfuerzos en la institución educativa para apoyar al estudiantado en el fortalecimiento de herramientas para la prevención y manejo del estrés como parte de un plan para disminuir problemáticas de salud, logrando con ello, el aumento de la motivación en la población estudiantil, lo cual puede repercutir en una disminución de la deserción y la repitencia.

La gestión de la educación debe ser promotora de una educación visionaria, en la cual el ser humano reciba aprendizajes para la vida mediante el desarrollo de destrezas, competencias y habilidades que les permitan sobrellevar exitosamente problemáticas como el estrés escolar.

Existen diversas técnicas para el manejo y prevención del estrés que, como profesionales en orientación en conjunto con la administración de los centros educativos, es fundamental conocerlas y propiciar su implementación, partiendo de las necesidades presentadas en las poblaciones con las cuales se labore, con la finalidad de fortalecer en ellas factores protectores del distress y reforzadores del eustress para mejorarles su calidad de vida, rendimiento laboral, personal y educativo.

Los participantes del estudio perciben el estrés como algo nocivo para la salud, lo cual les dificulta disfrutar plenamente de las tareas a realizar, les genera más agotamiento, disminución de la motivación y por ende, menos disfrute del ejercicio de sus quehaceres; sin embargo, la teoría muestra como éste, puede ser utilizado como impulso para alcanzar los propósitos obtenidos si se canalizan adecuadamente las energías, aspecto trascendental de trabajar, con la finalidad de prevenir problemas de deserción y aumentar la salud de las personas.

Existe una inminente necesidad en la población del estudio de gestionar, en forma conjunta dirección y Departamento de Orientación, planes de trabajo que incluyan la implementación de técnicas para la prevención y el manejo del estrés, pues la población estudiantil menciona sentirse agotada de las presiones de la vida cotidiana, especialmente de las sobrecarga de

Licencia Creative Commons

Atribución-No-Comercial SinDerivadas (cc) (†) $\$$ 3.0 Costa Rica. 
Rev. Gestión de la Educación, Vol. 4, N² 2, [131-149], ISSN: 2215-2288, julio-diciembre, 2014 DOI: http://dx.doi.org/10.15517/rge.v4i2.15148

URL: http://revistas.ucr.ac.cr/index.php/gestedu
Gestión 용 Educación

Escuela de Administración Educativa

funciones que tienen, al ser padres o madres de familia, trabajadores y estudiantes. Ante lo cual, es necesario recordar que una de las finalidades de los sistemas educativos es formar personas con competencias y habilidades personales y sociales, donde se vean beneficiados en su desarrollo integral y calidad de vida; por ende, la necesidad de trabajar esta temática.

La familia, el trabajo y el estudio son aspectos que permutan y afectan mutuamente, por lo tanto, si se quiere ofrecer una educación de calidad, es importante trabajarlos en conjunto, recordando que cada cual tiene factores estresantes propios, los cuales van a responder, muchas veces, a características particulares de las personas y el contexto en donde se desarrolla, haciéndose necesario el acompañamiento de profesionales para lograr superar los retos y lograr mayores niveles de eustress. Las técnicas para el manejo y prevención del estrés permiten mitigar el efecto negativo producido por los múltiples agentes estresores de la sociedad, por lo cual la importancia de propiciar la guía de su uso en poblaciones tan susceptibles como la del estudio, para contribuir a mejorar su calidad de vida.

Las estrategias para el manejo del estrés, por parte de las personas del estudio, se vinculan con la necesidad de acompañamiento de personas significativas para ellas, como lo es la familia y el grupo de pares, denotándose la importancia del reforzamiento social como ente estimulador de salud física-mental.

\section{Referencias}

Barrantes, R. (2006). Investigación. Un camino al conocimiento. Un enfoque cuantitativo y cualitativo. San José, Costa Rica: EUNED.

Barraza, A. (2007). El campo de estudio del estrés: del Programa de Investigación EstímuloRespuesta al Programa de Investigación Persona-Entorno. Revista Internacional de Psicología, 8(2), 1-30. ISSN 1818-1023. Recuperado en: http://psicologiarevista.99k.org/ El\%20campo\%20de\%20estudio\%20del\%20estres.pdf

Barraza, A. (2010). La relación persona-entorno como fuente generadora de estrés académico. Revista Internacional de Psicología, 11(1), 1-11. ISSN 1818-1023. Recuperado en: http://psicologiarevista.99k.org/La\%20relacion\%20persona\%20entorno\%20como\%20 fuente\%20generadora\%20de\%20estres\%20academico.pdf

Bautista, M. y Ré, R. (2007). La vida sin distrés. Argentina: San Pablo.

\begin{tabular}{|c|c|c|}
\hline (c) $(7) \Theta$ & $\begin{array}{l}\text { Licencia Creative Commons } \\
\text { Atribución-No-Comercial SinDerivadas } \\
\text { 3.0 Costa Rica. }\end{array}$ & \\
\hline & $\begin{array}{l}\text { Wilbert Porras Quirós, Marianela Araya Marín y } \\
\text { Laura Fallas Fallas }\end{array}$ & Número publicado el 1 de julio del 2014 \\
\hline
\end{tabular}


Berrío, N. y Mazo, R. (2011). Estrés académico. Revista de Psicología Universidad de Antioquia, 3(2), 67. ISSN 2256-1102. Recuperado de http://aprendeenlinea.udea.edu.co/revistas/index.php/ psicologia/article/view/11369/10646

Carrillo, A. (2010). Cromoterapia. México: Universidad Autónoma del Estado de Morelos.

Correa de Urrea, A., Álvarez, A. y Correa, S. (s.f.). La gestión educativa: un nuevo paradigma. Recuperado de http://virtual.funlam.edu.co/repositorio/sites/default/files/6lagestioneduc ativaunnuevoparadigma.pdf

De Mézerville, G. (2004). Ejes de la salud mental: Las crisis del desarrollo y los desajustes transicionales. México: Trillas.

De Mézerville, G. (2012). Técnicas para reducir el estrés. Recuperado de http://www.psicopedagogia. com/tecnicas-reducir-estres

Fernández, L. (2010). Modelo de intervención en crisis. En busca de la resiliencia personal. Recuperado de http://www.luriapsicologia.com/mediateca/TRAB\%20MODELO\%20DE\%20 INTERVENCION\%20EN\%20CRISIS\%20-Lourdes\%20Fernandez.pdf

Fondo de las Naciones Unidad para la Infancia (UNICEF). (s.f.). Educación practica para la vida. Recuperado de http://www.unicef.org/spanish/education/index focus lifeskills.html

Landy, F. y Conte, J. (2005). Psicología Industrial: Estrés y salud en el trabajo, teorías sobre el estrés, reducción y manejo del estrés. México: Mc Graw-Hill Interamericana.

McDonald, A. (2001). The prevalence and effects of test anxiety in school children. Educational Psychology, 21, 89-101. Recuperado de http://www.tandfonline.com/doi/abs/10.1080/01443 410020019867\#preview

Ministerio de Educación Pública de Costa Rica. (2008). El desarrollo y el estado de la cuestión sobre el aprendizaje y la educación de adultos (AEA). Recuperado de http://www.unesco. org/fileadmin/MULTIMEDIA/INSTITUTES/UIL/confintea/pdf/National Reports/Latin\%20 America\%20-\%20Caribbean/Costa Rica.pdf

Licencia Creative Common .0 Costa Rica. 
Naranjo P., M. (2009). Una revisión teórica sobre el estrés y algunos aspectos relevantes de éste en el ámbito educativo. Revista de Educación [en línea], 33(2), 171-190. Recuperado de http:// redalyc.uaemex.mx/src/inicio/ArtPdfRed.jsp?iCve $=44012058011$

Pedagogía 300. (s.f.). Mandalas. El despertar de la conciencia planetaria. Recuperado de http:// www.pedagooogia3000.info/web/html/Despertar.pdf

Peraza, E. y Zaldívar, D. (2003). La Musicoterapia. Un nuevo enfrentamiento al estrés y la Hipertensión Arterial. Revista Cubana de Psicología, 20(1), 10-22. Recuperado de_http:// biblat.unam.mx/pt/revista/revista-cubana-de-psicologia/articulo/la-musicoterapia-unnuevo-enfrentamiento-al-estres-y-la-hipertension-arterial.

Pulido, M., Serrano, M., Valdés, E., Chávez, M., Hidalgo, P. y Vera, F. (2011). Estrés académico en estudiantes universitarios. Psicología y Salud, 21(1), 31-37. Recuperado de http://www. uv.mx/psicysalud/psicysalud-21-1/21-1/Marco-Antonio-Pulido-Rull.pdf

Roca, S. (s. f.). Musicoterapia. Recuperado de http://www.imsersomayores.csic.es/documentos/ documentos/uszheimer-musica-01.pdf

Rose, S. (2002). Guía del antiestrés. Barcelona: Parragón.

Sandín, B. (2002). El estrés: un análisis basado en el papel de los factores sociales. Revista Internacional de Psicología Clínica y de la Salud, 3(1), 141-157. Recuperado de http://www. aepc.es/ijchp/articulos pdf/ijchp-65.pdf

Zeidner, M. (1998). Test anxiety: the state of the art. New York: Plenum Press. 